\title{
ASSESSMENT OF OLFACTORY DYSFUNCTION IN PARKINSON'S DISEASE PATIENTS
}

Akhilesh Kumar Singh *1, Bal Krishana ${ }^{2}$, Meena Gupta ${ }^{3}$.

${ }^{{ }_{1}}$ Assistant Professor, Department of Physiology, Andaman \& Nicobar Islands Institute of Medical Sciences, Port Blair, India.

2 Professor, Department of Physiology, Maulana Azad Medical College, New Delhi, India.

${ }^{3}$ Professor, Department of Neurology, Govind Ballabh Pant Hospital, New Delhi, India.

\section{ABSTRACT}

Parkinson's disease (PD) is a progressive neurodegenerative disorder of dopaminergic neurons in the substantia nigra pars compacta, its incidence and prevalence increases with age. Autonomic, cognitive, and sensory symptoms occur frequently in PD along with motor disturbances. The disturbances of olfaction are major, but often overlooked by PD patients. The present study was conducted on 30 patients of PD and 30 age matched controls, of the age group 40-70 years. Besides motors symptoms, history of non-motor symptoms like sleep disturbances, forgetfulness, constipation and urinary problem was taken, though these problems are also associated with old age. 18 patients gave history of forgetfulness. 19 patients had some sort of sleep disturbances. 18 patients had history of constipation and 17 patients gave history of urinary problem. History of olfactory loss or altered olfaction, altered taste sensation was also taken. 11 patients were aware of their olfactory loss or altered olfaction. Out of these 11 patients, 2 patients had complete bilateral anosmia, 2 patients had right sided complete anosmia and 5 patients had history of hyposmia. 21 patients had no history of olfactory loss or altered olfaction. 5 patients had also altered taste sensation. Identification of earlier clinical markers is paramount for success in putative preventive treatments. Besides olfactory dysfunction, the other clinical markers in PD are: upper limb kinematics behavior, cognition impairment, depression, sleep disorders, and micrographia. Identifying subjects with an increased risk of developing PD may contribute to the development of neuroprotective treatment strategies, as a preclinical diagnosis would allow neuroprotective agents to be administered earlier in the disease process.

KEY WORDS: Parkinson's disease (PD), Olfactory dysfunction, Non-motor symptoms.

Address for correspondence: Akhilesh Kumar Singh, Assistant Professor, Department of Physiology, Andaman \& Nicobar Islands Institute of Medical Sciences, Port Blair, India.

E-Mail: dr.akhi@yahoo.co.in

\begin{tabular}{|c|c|c|}
\hline \multicolumn{3}{|c|}{ Online Access and Article Informtaion } \\
\hline \multirow{2}{*}{$\begin{array}{l}\text { Quick Response code } \\
\text { DOI: } 10.16965 / \text { ijims.2017.116 }\end{array}$} & \multicolumn{2}{|c|}{$\begin{array}{l}\text { International Journal of Integrative Medical Sciences } \\
\text { www.imedsciences.com }\end{array}$} \\
\hline & $\begin{array}{l}\text { Received: 15-11-2017 } \\
\text { Reviewed: 16-11-2017 }\end{array}$ & $\begin{array}{l}\text { Accepted: 07-12-2017 } \\
\text { Published: 21-12-2017 }\end{array}$ \\
\hline Source of Funding: Self & \multicolumn{2}{|c|}{ Conflicts of interest: None } \\
\hline
\end{tabular}

\section{INTRODUCTION}

Parkinson's disease (PD) is an adult onset, gradually progressive, neurodegenerative disease of dopaminergic neurons in substantia nigra pars compacta, and their projections to striatal regions and is characterized by cardinal features including resting tremor, rigidity, bradykinesia and in more advance cases postural instability $[1,2]$. It is the second most common neurodegenerative disorder of the ageing brain with debilitating motor dysfunctions [3]. The main neuropathological feature of PD is the loss of neuromelanin containing dopaminergic neurons in the substantia nigra pars compacta (SNPC) and the presence of intra-cytoplasmic inclusions known as Lewy bodies [4], which leads to dopamine deficiency in the corpus striatum and substantia- 
-nigra (SN) [5]. This dopamine depletion leads to the development of motor abnormalities like tremor at rest, rigidity, slowness of movements and postural instability in the patients of PD. At the time of clinical manifestation of the disease, $60 \%$ of the nigral dopaminergic neurons have been lost and the striatal content of dopamine has been reduced by $80 \%$. Significant amount of dopamine is also present in olfactory tubercle. Since dopamine has been found to be present in olfactory tubercle and Parkinsonism is a disease of decreased brain dopamine level, it is possible that a decrease in olfactory acuity may be associated with this syndrome [6].

$P D$ is primarily a disease affecting motor functions. Tremor is the most common initial symptom of PD [7]. Postural instability occurs in advanced cases of PD [8]. PD also affects cognitive and sensory processing. Of the sensory impairments associated with PD, olfactory impairments have received most attention and are an attractive potential biomarker as an early clinical sign for PD because of its high prevalence among PD patients as well as its ease of testing [9].

Olfactory dysfunction, whether measured by odor identification, recognition, or threshold, is associated with PD and may be the earliest sign [10]. PD patients exhibit impairments in odor detection, defects concerning the processing of olfactory information including the identification of odors, and discrimination of odors, and a reduced olfactory evoked response. This occurs so consistently that the olfactory impairments may have diagnostic value for PD [9].

Olfaction requires complex sensory-motor integration [9]. To date pathological changes in PD have been reported in various regions involved in olfactory function, including the anterior olfactory nucleus, the olfactory bulb, the periamygdaloid cortex, and the olfactory mucosa, as well as impairment in sniffing [11]. Olfactory bulb and tract are among the earliest brain regions affected by Lewy pathology. The finding of incidental Lewy bodies (ILB) in the brain of the patients having impaired olfaction but no motor symptoms or PD, provide evidence that olfactory dysfunction may predate the typical motor signs of PD [10].
Very few studies have been done from our subcontinent focusing on the prevalence of olfactory dysfunction in PD and its correlation with stage and duration of the disease, as this non-motor symptom has received little attention of both patients as well as clinicians. Hence we conducted this study to assess the olfaction in a selected group of patients with PD and to correlate it with stage and duration of the disease.

\section{MATERIALS AND METHODS}

This study was conducted in the Department of Physiology at Maulana Azad Medical College and Department of Neurology Govind Ballabh Pant Hospital, New Delhi.

Selection of Subjects: The study comprised 30 patients of PD selected from Department of Neurology, Govind Ballabh Pant Hospital, and 30 sex and age matched controls, who were not suffering from PD. These controls were selected from amongst the staff of Maulana Azad Medical College and associated Lok Nayak Hospital and Govind Ballabh Pant Hospital, New Delhi and the general population. Procedures for all examination were in accordance with the institutional guidelines and approved by an institutional review committee. An informed written consent was taken from all study participants. All participants agreed to undergo the proposed tests.

\section{Inclusion criteria}

1. Patients of PD (age $40-70 \mathrm{yr}-\mathrm{s}$ ).

2. Persons without PD (age $40-70$ yrs).

3. Patients with normal retina and optic nerve.

\section{Exclusion criteria}

Cases of Parkinsonism related to

1. Progressive supranuclear palsy

2. Multiple system atrophy

3. Drug induced parkinsonism

4. Post encephalitic parkinsonism

5. Post traumatic parkinsonism.

6. Olfactory dysfunction due to causes other than PD.

\section{Study Design}

Control group: Age and sex matched controls that were not suffering from PD or illnesses known to be associated with olfactory dysfunction and free from the diseases to be 
excluded from the study group were selected. Subjects exhibiting any evidence of dementia or atypical parkinsonian feature were excluded. Controls with a family history of PD were also excluded. All the subjects included were literate with a minimum education up to fifth standard.

Parkinson's Disease Study Group: Patients suffering from PD were enlisted for the study after obtaining an informed written consent. Patients of PD were matched for age and sex with normal controls. All were community dwelling and all were living in their own homes. Out of total thirty patients, 23 were male and 7 were females. The mean age of the PD patients was $58.10 \pm 9.90$ years with age range of $40-70$ years. A detailed history regarding onset of symptoms and their duration was obtained. History regarding non motor symptoms of PD like urinary problems, bowel dysfunction, forgetfulness, and sleep related problems was also obtained. The duration of the disease ranged from 1-15 years with an average duration of $5.42 \pm 3.68$ years. Diagnosis of PD was made in Neurology clinic, Department of Neurology, Govind Ballabh Pant Hospital, New Delhi. The diagnosis was based on patient history and physical examination employing the diagnostic criteria outlined by UK PDS Brain Bank diagnostic criteria.

\section{UK PDS Brain Bank diagnostic criteria includes} [12]:

STEP 1 - Diagnosis of Parkinsonian syndrome Bradykinesia plus at least one of the following
(a)
Muscular rigidity
(b) Rest tremor
(c) Postural instability

STEP 2 - Exclusion criteria including
(a) History of repeated strokes
(b) History of repeated head injury
(c) History of definitive encephalitis

STEP 3 - Supportive prospective criteria (at least three required)
(a) Unilateral onset
(b) Rest tremor present
(c) Evidence of progression
(d) Persistent asymmetry
(e) Excellent response to L-dopa
(f) Severe L-dopa-induced chorea
(g) L-dopa response for $5+$ years
(h) Clinical course of $10+$ years

A comprehensive and standardized neurological examination that included the Unified Parkinson's Disease Rating Scale (UPDRS) [13] was done by a neurologist in the Department of Neurology, Govind Ballabh Pant Hospital. The presence and severity of parkinsonian symptoms were determined and quantified by means of the motor section (part III) of the Unified Parkinson's Disease Rating scale.

\section{Unified Parkinson's disease Rating scale (UPDRS) [13]}

The UPDRS is a composite scale consisting of four parts.

1. Mentation, behavior and mood.

2. Activities of daily living (determine for on/ off).

3. Motor examination.

4. Complications of therapy (in the past week). All patients were categorized according to Hoehn and Yahr criteria [11].

\section{Modified Hoehn and Yahr staging}

Stage 0- no sign of disease.

Stage 1- unilateral disease.

Stage 1.5- unilateral plus axial involvement.

Stage 2- bilateral disease, without impairment of balance.

Stage 2.5- mild bilateral disease with recovery on pull test.

Stage 3- mild to moderate bilateral disease; some postural instability; physically independent.

Stage 4- $\quad$ severe disability; still able to walk or stand unassisted.

Stage 5- wheel chair bound or bed ridden, unless aided.

The patients were mostly of stage 2 and 3 on the Hoehn and Yahr staging of PD. History concerning motor dysfunction, age at diagnosis, first symptoms and signs and onset, current and past smell problems or any change in their ability to smell things and whether it was responsive to antiparkinsonian or other drugs, current and past medications, and current and past smoking habits was obtained. An interview was performed to establish any family history of PD and to confirm medical history. History of nasal surgery or sinus surgery, thalamic surgery, nasal injury or severe head trauma, obstructive lung 
disease or allergies causing nasal congestion was also asked as these can affect olfaction and have to be excluded. No patient gave past history of head trauma or nasal injury. 21 patients were non smokers and 9 patients gave history of smoking. Patients with dementia, acute or chronic sinusitis, recent upper respiratory tract infection, diabetes mellitus, alcoholism, clinical evidence of liver dysfunction or other illness that might conceivably result in decreased olfactory acuity were not included in this study. All patients were taking dopaminergic replacement medication and were tested in the "on" condition (i.e. typically in the middle of the time window between drug administrations), but these probably donot affect the ability to smell [14]. The patients with arterial hypertension, diabetes were excluded.

Tests for Olfactory Function: Several patients who present with complaints of anosmia or hyposmia actually have normal function relative to their peer. Others are unaware of their dysfunction. In the case of PD, $60-90 \%$ of patients have a demonstrable olfactory loss, yet less than $15 \%$ are aware of the problem until being tested [15]. Olfactory dysfunction has been shown to help in the differentiation of PD from other causes of Parkinsonism, to the extent that in evaluating a patient with parkinsonian sign but preserved olfaction, the diagnosis of early PD should be reconsidered [16].

Electrophysiological, psychophysical, and psycho-physiological tests are available for assessing smell function [15]. The most practical are psychophysical tests of odor identification and detection. Despite the fact that bilateral testing detects most clinically meaningful cases of olfactory dysfunction, unilateral testing can detect deficits that are not ordinarily recognized. In general, bilateral tests measure the functioning of the better side of the nose [15]. To assess unilateral function, the naris contralateral to the tested side is occluded to prevent or minimize crossing of inhaled or exhaled air at the rear of the nasopharynx to the opposite side (so called retronasal stimulation).

Olfactory function was assessed by means of odor detection, odor discrimination, and an odor identification task. Tests of olfaction are commo- nly based on total olfactory performance after exposure to multiple odors [11]. The nose was checked for patency and all patients had intact nasal passages.

Test Material: Glass vials containing the stimuli were used; one screw cap had to be removed when testing. The vials had a second porous top allowing sniffing and perception of the odor while preventing any spillage of the odorous substance. This material was thus suitable for handling by patients with motor disturbances. The olfactory stimuli consisted of odorous substances which were:

(a) Non irritant

(b) Undetected via intranasal trigeminal afferents

(c) Whose smell was familiar to the subjects and readily identifiable by them

(d) That they produce no untoward reactions or discomfort to the subjects

Procedure: One side of the nose was tested for olfaction at a time. The other side was occluded. One vial containing the odorous material was asked to sniff at a time and the subject was instructed not to see the material inside the vial. For that subject was asked to close his eyes while sniffing. Subject was also asked to identify the odors and remember them so as to subsequently discriminate it from previous and next odorous substances. The subject then answered some questions from the questionnaire which included, "can you detect it", "can you identify it", "can you discriminate it from previous one" and "can you recall the previous odor". The answers were recorded as yes or no. Similar procedure was repeated for the other side of the nose also.

\section{OBSERVATIONS AND RESULTS}

In the present study, 30 patients of PD were persuaded to enroll in the study along with a group of 30 sex and age matched controls, who were not suffering from PD. The study was aimed at assessing the olfactory dysfunction of these subjects and to compare the results between the group of PD and controls. The following observations were recorded in 30 normal controls and 30 patients of PD. The PD patients were recruited from neurology clinic, Department of Neurology, G.B. Pant Hospital Delhi. 
Demographic data of the study population: As shown in table 1, out of 30 controls, 23 were male and 7 were female and out of 30 PD patients 23 were male and 7 were females.

Table 1: Age wise distribution in the two groups of subjects compared.

\begin{tabular}{|c|c|c|c|}
\hline S.No. & $\begin{array}{c}\text { Age range } \\
\text { (years) }\end{array}$ & Controls & PD patients \\
\hline 1 & $40-50$ & 8 & 8 \\
\hline 2 & $51-60$ & 9 & 8 \\
\hline 3 & $61-70$ & 13 & 14 \\
\hline
\end{tabular}

It can be seen from the Table-1 that majority of the patients in both the groups were in age range of $61-70$ years.

The mean age of the controls was $57.67 \pm 8.45$ years with age range of $40-70$ years. The mean age of the PD patients was $58.10 \pm 9.90$ years with age range of $40-70$ years. The demographic and anthropometric data of controls and PD patients are shown in the Table-2.

Table 2: Demographic and anthropometric profile of controls and PD patients compared.

\begin{tabular}{|c|c|c|c|}
\hline S.No. & $\begin{array}{c}\text { Controls } \\
\text { Mean } \pm \text { SD } \\
(\mathbf{n}=\mathbf{3 0})\end{array}$ & $\begin{array}{c}\text { PD patients } \\
\text { Mean } \pm \text { SD } \\
(\mathbf{n}=\mathbf{3 0})\end{array}$ \\
\hline 1 & $\begin{array}{c}\text { Age (Years) } \\
\text { Range }\end{array}$ & $\begin{array}{c}57.63 \pm 8.60 \\
40-70\end{array}$ & $\begin{array}{c}58.10 \pm 9.90 \\
40-70\end{array}$ \\
\hline 2 & M:F & $23: 07$ & $23: 07$ \\
\hline 3 & Weight (Kg) & $63.10 \pm 7.64$ & $66.10 \pm 5.29$ \\
\hline 4 & Height (Cms) & $160.07 \pm 6.88$ & $162.07 \pm 6.84$ \\
\hline 5 & $\begin{array}{c}\text { Duration of } \\
\text { disease } \\
\text { (Years) }\end{array}$ & -- & $5.42 \pm 3.68$ \\
\hline & H \& Y stage & & \\
& 1 & - & 14 \\
& 2 & & 14 \\
\hline
\end{tabular}

From the Table-2 it can be seen that both controls and PD patients were age and sex matched. In both controls and PD patients, 23 were males and 7 were females.

The mean duration of disease in PD patients was $5.42 \pm 3.68$ years. Most of the PD patients were in stage 2 or stage 3 of the Hoehn and Yahr staging of the PD.

Out of 30 PD patients, 29 patients were on dopaminergic therapy. The mean current daily Syndopa dosage in PD patients was $382.3 \pm 198.25 \mathrm{mg}$ and the mean duration of Syndopa treatment was $3.74 \pm 2.68$ years.

Out of 30 PD patients, 2 patients had complete bilateral anosmia and 2 patients had right sided complete anosmia. 5 patients had history of hyposmia, while 21 patients had no history of hyposmia. None of them had any improvement in olfaction after Syndopa or antiparkinsonian treatment.

Olfactory score parameters: The Mean \pm SD scores of the different olfactory processing tasks performed on both sides of the nose are as shown in Table -3.

Table 3: The Mean \pm SD scores of different olfactory processing tasks in the two groups of subjects compared.

\begin{tabular}{|c|c|c|c|c|}
\hline S.No. & Olfactory processing tasks & $\begin{array}{c}\text { Controls } \\
\text { Mean } \pm \text { SD } \\
(\mathbf{n}=\mathbf{3 0})\end{array}$ & $\begin{array}{c}\text { PD patients } \\
\text { Mean } \pm \text { SD } \\
(\mathbf{n}=\mathbf{3 0})\end{array}$ & p-value \\
\hline 1 & Odor detection (L) & $10 \pm 0.0$ & $7.80 \pm 2.72$ & VHS \\
\hline 2 & Odor detection (R) & $10 \pm 0.0$ & $7.43 \pm 3.34$ & VHS \\
\hline 3 & Odor identification (L) & $9.77 \pm 0.43$ & $5.9 \pm 2.83$ & VHS \\
\hline 4 & Odor identification (R) & $9.77 \pm 0.43$ & $6.2 \pm 2.94$ & VHS \\
\hline 5 & Odor discrimination (L) & $10 \pm 0.0$ & $7.57 \pm 2.7$ & VHS \\
\hline 6 & Odor discrimination (R) & $10 \pm 0.0$ & $7.40 \pm 3.17$ & VHS \\
\hline 7 & Odor Recall (L) & $9.77 \pm 0.43$ & $5.37 \pm 2.84$ & VHS \\
\hline 8 & Odor Recall (R) $(\mathrm{l}$ & $9.77 \pm 0.43$ & $4.97 \pm 2.94$ & VHS \\
\hline 9 & Total Olfactory score (L) & $39.53 \pm 0.86$ & $26.63 \pm 10.27$ & VHS \\
\hline 10 & Total Olfactory score (R) & $39.53 \pm 0.86$ & $26.07 \pm 11.48$ & VHS \\
\hline
\end{tabular}

$\mathrm{L}=$ Left side of the nose, $\mathrm{R}=$ Right side of the nose, $\mathrm{VHS}$ $=$ Very highly significant $(p$-value $<0.001)$

It can be seen from the Table-3 that PD patients had lower olfactory scores as compared to the matched controls.

The Mean \pm SD total olfactory scores of left and right side of the nose in different subgroups of PD patients according to age have been compared in Table-4.

Table 4: The Mean \pm SD total left and right olfactory scores in different subgroups of PD patients according to age compared.

\begin{tabular}{|c|c|c|c|}
\hline S.No. & $\begin{array}{c}\text { Age groups } \\
\text { (in years) }\end{array}$ & $\begin{array}{c}\text { Total Olfactory } \\
\text { Score (L) } \\
\text { Mean } \pm \text { SD }\end{array}$ & $\begin{array}{c}\text { Total Olfactory } \\
\text { Score (R) } \\
\text { Mean } \pm \text { SD }\end{array}$ \\
\hline 1 & $40-50$ & $29.75 \pm 10.27$ & $31.0 \pm 9.01$ \\
\hline 2 & $51-60$ & $29.13 \pm 10.58$ & $27.0 \pm 11.48$ \\
\hline 3 & $61-70$ & $23.43 \pm 12.45$ & $22.71 \pm 12.88$ \\
\hline
\end{tabular}

$\mathrm{L}=$ Left side of the nose, $\mathrm{R}=$ Right side of the nose

It can be seen from the Table- 4 that the olfactory scores were decreasing with higher age group patients. The olfactory scores were lowest in age group 61-70 years.

It can also be seen from the table that the olfactory scores of left and right side of the nose 
of patients are not significantly different.

The Mean \pm SD total olfactory scores of left and right side of the nose with different durations of the disease have been compared in Table-5.

Table 5: The Mean \pm SD total olfactory scores of left and right side of the nose with different durations of the disease compared.

\begin{tabular}{|c|c|c|c|}
\hline S.No. & $\begin{array}{c}\text { Duration } \\
\text { of disease } \\
\text { in years }\end{array}$ & $\begin{array}{c}\text { Total Olfactory } \\
\text { Score (L) } \\
\text { Mean } \pm \text { SD }\end{array}$ & $\begin{array}{c}\text { Total Olfactory } \\
\text { Score (R) } \\
\text { Mean } \pm \text { SD }\end{array}$ \\
\hline 1 & $01-$ May & $28.10 \pm 9.10$ & $27.17 \pm 11.43$ \\
\hline 2 & $06-$ Oct & $26.12 \pm 12.58$ & $26.34 \pm 12.42$ \\
\hline 3 & Nov-15 & $27.89 \pm 11.23$ & $28.73 \pm 10.88$ \\
\hline
\end{tabular}

$\mathrm{L}=$ Left side of the nose, $\mathrm{R}=$ Right side of the nose

It can be seen from the Table-5 that left and right total olfactory scores are not significantly different with different duration of the disease.

The Mean \pm SD total olfactory scores of left and right side of the nose with different stages of Hoehn and Yahr staging of the disease have been compared in Table- 6 .

Table 6: The Mean \pm SD left and right total olfactory scores of different stages of PD compared.

\begin{tabular}{|c|c|c|c|}
\hline S.No. & $\begin{array}{c}\text { Hoehn and } \\
\text { Yahr stage }\end{array}$ & $\begin{array}{c}\text { Total Olfactory } \\
\text { Score (L) } \\
\text { Mean } \pm \text { SD }\end{array}$ & $\begin{array}{c}\text { Total Olfactory } \\
\text { Score (R) } \\
\text { Mean } \pm \text { SD }\end{array}$ \\
\hline 1 & 1 & $27.87 \pm 11.25$ & $29.14 \pm 10.55$ \\
\hline 2 & 2 & $28.86 \pm 10.85$ & $27.23 \pm 11.41$ \\
\hline 3 & 3 & $29.38 \pm 10.45$ & $27.76 \pm 11.36$ \\
\hline
\end{tabular}

$\mathrm{L}=$ Left side of the nose, $\mathrm{R}=$ Right side of the nose

It can be seen from the Table- 6 that left and right total olfactory scores are not significantly different with different stages of the disease.

The Mean \pm SD total olfactory scores of left and right side of the nose in male and female patients of PD have been compared in the Table7.

Table 7: The Mean \pm SD total olfactory scores of left and right side of the nose in male and female patients of PD compared.

\begin{tabular}{|c|c|c|}
\hline Sex & $\begin{array}{c}\text { Total Olfactory } \\
\text { Score (L) } \\
\text { Mean } \pm \text { SD }\end{array}$ & $\begin{array}{c}\text { Total Olfactory } \\
\text { Score (R) } \\
\text { Mean } \pm \text { SD }\end{array}$ \\
\hline Male & $26.39 \pm 12.40$ & $25.30 \pm 12.84$ \\
\hline Female & $28.43 \pm 11.36$ & $28.57 \pm 9.21$ \\
\hline
\end{tabular}

$L=$ Left side of the nose, $R=$ Right side of the nose

It can be seen from the Table-7 that the female patients have better total score as compared to male patients.

\section{DISCUSSION}

$P D$ is a progressive neurodegenerative disorder of dopaminergic neurons in the substantia nigra pars compacta. It is the most common movement disorder in middle or late life and its incidence and prevalence increase with age. The cause is unknown, but growing evidence suggests that it may be due to a combination of environmental and genetic factors. It is regarded primarily as a disorder of the motor system with bradykinesia, rigidity, tremor, gait and postural changes in advanced cases as its main features. However, autonomic, cognitive, and sensory symptoms frequently occur and PD has an impact on sensation, perception, cognition, sleep and emotional functioning [17]. The nonmotor features include mood disturbance, forgetfulness, disrupted sleep, depression, micrographia, difficulty in writing, olfactory dysfunction, urinary problems and constipation. Disturbances of olfaction are major, but often overlooked deficits found in PD.

The present study was conducted on 30 patients of PD and 30 controls, of the age group 40-70 years. PD patients and controls were matched for age and sex. Besides motors symptoms, history of non-motor symptoms like sleep disturbances, forgetfulness, constipation and urinary problem was taken, though these problems are also associated with old age. 18 patients gave history of forgetfulness. 19 patients had some sort of sleep disturbances. 18 patients had history of constipation and 17 patients gave history of urinary problem. History of olfactory loss or altered olfaction, altered taste sensation was also taken. 11 patients were aware of their olfactory loss or altered olfaction. Out of these 11 patients, 2 patients had complete bilateral anosmia, 2 patients had right sided complete anosmia and 5 patients had history of hyposmia. 21 patients had no history of olfactory loss or altered olfaction. 5 patients had also altered taste sensation.

In our study, four different olfactory processing tasks were performed. These tasks included: odor detection, odor identification, odor discrimination and odor recall. The mean total olfactory score in PD patient was $26.35 \pm 10.88$, while in controls, it was $39.26 \pm 1.23$. The data 
of olfactory test in our study revealed a defect in olfactory performance in PD patients in agreement with other previous studies done by Doty et al. (1988), Double et al. (2003), Katzenschlager et al. (2004), Ross et al (2008), Kranick et al. (2008) and it effectively discriminated between PD patients and control subjects. The odor deficit was not confined to any particular odor. In our study, $77 \%$ PD patients (23 patients) had olfactory dysfunction which is similar to the findings of earlier study done by Double et al. (2003) on olfactory dysfunctions in PD patients [18]. Double et al. conducted study on 49 patients with PD with mean age 68.8 years. The olfactory dysfunction in their study was tested by means of Brief Smell Identification Test (B-SIT). In their study $82 \%$ (40 patients) have abnormal olfactory function. Though the basis of olfactory dysfunction in PD is unknown, some hypotheses have been proposed to explain the olfactory dysfunction of Parkinson's disease. These hypotheses [19] are: First, the olfactory pathways could be impaired directly by environmental agents etiologically related to PD. Second, the underlying disease process decreases the resistance of the olfactory system to viral or environmental agents which cause destruction of the olfactory pathways. Third, sectors of the olfactory system are predisposed destruction from degenerative or neuropathological processes, which relate to an underlying disease process associated with PD.

In our study, 4 of 30 patients (17\%) had anosmia, of which 2 patients had complete bilateral anosmia and 2 patients had right sided complete anosmia. In the study conducted by Doty et al. [19] on 93 PD, 13\% had anosmia. In another study conducted by Ward et al. (1983) on 46 PD patients, 7 patients (17\%) had anosmia. In our study, 15 of 21 patients had no history of olfactory loss or altered olfaction. Our study showed that $71 \%$ of patients ( 15 of 21 patients having no history of olfactory loss or altered olfaction) were unaware of their smell disorder on a simple yes or no rating scale before the formal testing, and those who were aware of it, had significantly lower scores. This data of our study matches to the study done by Doty et al. (1988), in which $72 \%$ of the patients were unaware of their olfactory deficits before formal testing.
The data of our study suggest impairment in all the olfactory processing tasks in PD. The mean odor detection score in PD patients was $7.61 \pm$ 3.03 , while in the controls, it was $9.93 \pm 0.25$; the mean odor identification score in patients was $6.05 \pm 2.89$, while in controls, it was $9.77 \pm$ 0.46 ; the mean odor discrimination score in patients was $7.49 \pm 2.95$, while in controls, it was $9.93 \pm 0.25$; mean odor recall score in patients was $5.17 \pm 2.89$, while in controls, it was $9.77 \pm 0.46$.

The present study shows that 57\% PD patients have decreased odor detection score. In a study conducted by Doty et al. (1988), 75\% PD patients were reported to have decreased odor detection sensitivity. They conducted study on 81 PD patients with mean age $65.7 \pm 10.1$. In their study, a single staircase, forced-choice odor detection threshold test was used to estimate basal detection sensitivity to phenyl ethyl alcohol. The percentage of patients having lower odor detection score was more in their study because of more number of patients in their study and also because of more simplicity of task in our study. The patients in our study were being able to detect odor even if they were unable to identify it correctly.

The data in our study show that 24 of 30 patients (80\%) had lower olfactory identification score. In the same study conducted by Doty et al. (1988), using University of Pennsylvania Smell Identification Test (UPSIT), they reported 73 of 81 patients (90.1\%) UPSIT scores lower than their matched controls (20.2 \pm 4.7 in PD patients and $35 \pm 3.6$ in controls). ${ }^{19}$ The percentage of patients having lower identification was more in their study because they used 40 odors, while in our study, 10 odors were used.

In our study, 18 of 30 patients (60\%) had lower odor discrimination score. In a study conducted by Potagas et al. (1998), 50 patients of PD with mean age $66.1 \pm 9.4$ were selected and odor discrimination task was performed using a vial containing a chemical substance (iso-amylacetate). The vial containing the chemical substance was presented to the subject who was instructed to sniff and remember the odor so as to subsequently discriminate it from two other odors. In their study, 37 of 50 (72\%) patients had 
lower odor discrimination test score. The reason for this difference may be that we used common household odors for our olfactory task.

In our study, there was no statistically significant difference in total olfactory score between left and right side of the nose (Total olfactory score on left side was $26.63 \pm 10.27$, while in right side it was $26.07 \pm 11.48$ ) which is in agreement with the study conducted by Doty et al. (1992). In their study, Doty et al. (1992) selected 40 patients of PD and UPSIT test was performed on them. Left side UPSIT score of the patients in their study was $11.40 \pm 4.38$ and right side UPSIT score was $10.80 \pm 4.57$. Thus there was no statistically difference between UPSIT score of left side and right side in their study.

Our study did not show significant relation between olfactory dysfunction with stage of the PD. The mean total olfactory score of stage 1 patients was $28.12 \pm 9.08$; of stage 2 was 27.77 \pm 9.42 ; of stage 3 was $27.38 \pm 9.65$. Patients in all the three stages of PD showed lower olfactory scores. The study done by Doty et al. (1992) [15] also could not find relation between UPSIT score with stage of the patient. The study done by Double et al. (2003) [11] reported no interaction between B-SIT score and PD severity. In their study, 16 of 20 patients ( $80 \%$ ) of stage 1; 16 of 20 patients ( $80 \%)$; and 8 of 9 patients (89\%) had abnormal olfaction. One explanation for the lack of association between olfactory impairment and severity of disease is that olfactory deficits reach a maximum early in the course of the PD whereas motor signs continue to worsen through the later stages.

The data of study show that the olfactory dysfunction in our patients did not correlate to duration of the disease. The mean total olfactory score in patients with duration of disease up to 5 years was $27.85 \pm 10.07$; with duration of disease from 5 to 10 years was $26.23 \pm 12.31$; with duration of disease from 10 to 15 years was $28.73 \pm 9.89$. In the studies conducted by Doty et al. (1988) [15], Hawkes et al (1997) and Double et al. (2003) [11]; using UPSIT, no correlation of UPSIT score was reported with duration of the disease.

The data of our study show that olfactory deficits in patients are not particularly responsive to
L- dopa medication and duration of treatment. The mean total olfactory score of patients having duration of treatment up to 5 years was $26.68 \pm$ 10.23 , while those having duration of treatment more than 5 years was $27.76 \pm 9.63$. The study conducted by Double et al. (2003) demonstrated no interaction between Brief Smell Identification Test and duration of disease. The study conducted by Doty et al. (1992) reported no statistical difference of UPSIT score between untreated and treated PD patients. The UPSIT score in untreated patients was $11.40 \pm 4.38$, while in treated patients it was $11.15 \pm 4.39$. The lack of olfactory response to L-dopa treatment in PD patients may be due to irreversible loss of receptors, regional variation in penetration of L-dopa or a critical reduction in dopa-decarboxylase. This lack of olfactory response to L-dopa also proves that the olfactory dysfunction in PD is not only due to decreased dopamine level. The unresponsiveness to medication or duration of treatment also suggests that biochemical and pathological abnormalities other than nigrostriatal dopamine loss must be involved.

In the present study, the total olfactory score was compared in the subgroups of PD patients. The mean total olfactory score in the age group 40-50 was 30.35 \pm 9.55; in the age group 51-60, it was $28.07 \pm 10.17$; and in the age group 61 70 , it was $23.07 \pm 12.48$. The olfactory score was lower in the age group 61-70 as compared to the other two age groups and it may be due to age related changes in the olfaction.

In the present study, comparison of total olfactory score in male and female PD patients was also done. The mean olfactory score of male patients was $25.85 \pm 11.62$, while in female patients, it was $28.13 \pm 10.15$. In our study female patients have better total olfactory score than male patients which is in agreement with the study of Doty et al. (1992). In their study, women obtained higher UPSIT scores than men. The left and right UPSIT scores for male PD patients were $10.23 \pm 4.03$ and $10.00 \pm 4.16$ respectively; while in female patients the left and right UPSIT scores were $13.78 \pm 4.35$ and $13.22 \pm 4.68$ respectively.

In our study, smokers as well as nonsmokers were also included. 10 control subjects gave 
history of smoking currently or in the past, while in PD patients 9 gave history of smoking currently or in the past. Out of 16 PD patients having markedly lower olfactory score, 5 were smokers and remaining 11 were nonsmokers. The olfactory scores of the control group in our study were not significantly affected by their smoking behavior. The diminished olfactory score in nonsmokers shows that the olfactory dysfunction in PD is due to some other reasons also and smoking alone cannot explain the olfactory dysfunction in PD patients.

\section{CONCLUSION}

Identification of earlier clinical markers is paramount for success in putative preventive treatments. Besides olfactory dysfunction, the other clinical markers in PD are: upper limb kinematics behavior, cognition impairment, depression, sleep disorders, and micrographia. Identifying subjects with an increased risk of developing PD may contribute to the development of neuroprotective treatment strategies, as a preclinical diagnosis would allow neuroprotective agents to be administered earlier in the disease process.

\section{REFERENCES}

[1]. Sammi A, Nutt JG, Ransom BR. Parkinson's disease. Lancet 2004; 363: 1783-93.

[2]. Ponsen MM, Stoffers D, Booij J, Berthe LF, Wolters EC, Berendse HW. Idiopathic Hyposmia as a Preclinical Sign of Parkinson's Disease. Ann Neurol 2004; 56: 173-81.

[3]. George JL, Moses D, Wilkins S, Bush Al, Cherny RA Finkelstein DI. Targeting the Progression of Parkinson's Disease. Curr Neuropharmacol 2009; 7 (1): 9-36.

[4]. Stocchi F, Olanow C.W. Neuroprotection in Parkinson's disease clinical trials. Ann Neurol 2003; 53(suppl 3): S87- S99.

[5]. Poirier LJ, Sourkes TL. Influence of the substantia nigra on the catecholamine content of the striatum. Brain 1965; 88: 181-92.

[6]. Ansari KA, Johnson A. Olfactory function in patients with Parkinson's disease. J Chron Dis 1975; 28: 4937.

[7]. Navan P, Findley LJ, Jeffs JAR, Pearce RKB, Bain PG. Randomised, double-blind, 3-month parallel study of the effects of pramipexole, pergolide, and placebo on Parkinsonian tremor. Mov Disord 2003; 18(11): 1324-31.

[8]. Dimitrova D, Horak FB, Nutt JG. Postural Muscle Responses to Multidirectional Translations in Patients with Parkinson's disease. J Neurophysiol 2004; 91: 489-501.
[9]. Sobel N, Thomasan ME, Stappen Iris, Tanner MC, Tetrud JW, Bower JM, et al. An impairment in sniffing contributes to the olfactory impairment in Parkinson's Disease. PNAS 2001; 98 (7): 4154-9.

[10]. Ross GW, Abott RD, Petrovitch H, Tanner CM, Davis DG, Nelson J, et al. Association of Olfactory Dysfunction with Incidental Lewy Bodies. Mov Disord 2006; 21 (12): 2062-7.

[11]. Double KL, Rowe DB, Hayes M, Chan DKY, Blackie J, Corbett $A$, et al. Identifying the Pattern of Olfactory deficits in Parkinson Disease Using the Brief Smell Identification Test. Arch Neurol 2003; 60: 545-9.

[12]. Hughes AJ, Daniel SE, Kilford L, Lees AJ. Accuracy of clinical diagnosis of idiopathic Parkinson's disease: a clinico-pathological study of 100 cases. J Neurol Neurosurg Psychiatry 1992; 55 (3): 181-4.

[13]. Ross GW, Petrovitch H, Abbott RD, Tanner CM, Popper J, Masaki K, et al. Association of Olfactory Dysfunction with Risk For Future Parkinson's Disease. Ann Neurol 2008; 63: 167-73.

[14]. Regan, D. Human Brain Electrophysiology. Evoked Potentials and Evoked Magnetic Fields in Science and Medicine. Elsevier, Amsterdam, 1989: 677pp.

[15]. Doty RL. The Olfactory system and its Disorders. Semin Neurol 2009; 29 (1): 74-81.

[16]. Ansari KA, Johnson A: Olfactory function in patients with Parkinson's disease. J chronic Dis 1975; 28: 493-97.

[17]. Mizuno Y, Hattori N, Kubo S, Sato S, Nishioka K, Hatano $T$ et al. Progress in the pathogenesis and genetics of Parkinson's disease. Philos Trans R Soc Lond B Biol Sci 2008;363(1500): 2215-27.

[18]. DeLong MR, Wichmann T. Circuits and Circuit Disorders of the Basal Ganglia. Arch Neurol 2007;64: 20-4.

[19].Russchen FT, Bakst I, Amaral DG, Price JL. The amygdalostriatal projection in the monkeys. An anterograde tracing study. Brain Res 1985; 329 : 241-57.

[20]. Parent A. Extrinsic connections of the basal ganglia. Trends Neurosci 1990; 13: 254-8.

[21]. Albin RL, Young AB, Penney JB. The functional anatomy of basal ganglia disorders. Trends Neurosci 1989; 12: 366-75.

[22]. Parent A, Hazrati LN. Functional anatomy of the basal ganglia. I. The cortico-basal ganglia-thalamocortical loop. Brain Res Rev 1995; 20: 91-127.

How to cite this article: Akhilesh Kumar Singh, Bal Krishana, Meena Gupta. ASSESSMENT OF OLFACTORY DYSFUNCTION IN PARKINSON'S DISEASE PATIENTS. Int J Intg Med Sci 2017;4(7):528-536. DOI: 10.16965/ijims.2017. 116 